\title{
Bioengineering of malaria vectors, anopheles mosquitoes (Diptera: Culicidae) as a control strategy: An overview
}

\section{Kayode David Ileke ${ }^{1}$ and Isaac Omotayo Olabimi ${ }^{2}$}

${ }^{1}$ Applied Entomology Unit. Department of Biology. School of Science. Federal University of Technology. P.M.B. 704. Akure. Ondo State. Nigeria. Email: kdileke@futa.edu.ng.

${ }^{2}$ Enviromental Biology and Public Health Unit. Department of Biology. School of Science. Federal University of Technology. P.M.B. 704. Akure. Ondo State. Nigeria.

\begin{abstract}
Disease in any form is undesirable in any human population. Malaria is a communicable disease that is vectored by female Anopheles mosquitoes. It is the leading vector transmitted disease in terms of the number of morbidity and mortality accounting for over 200 million cases annually. Several control measures have been employed by man over the years to control the vector which will in turn lead to the control of the diseases with the popular ones involving the use of insecticidal nets and indoor residual spraying of insecticides. However, these control measures have their various pitfalls. The use of genetically modified mosquitoes (GMMs) through bioengineering may be a promising method of reducing malaria vector population in our environment. This process involves population replacement technique (PRT) and population suppression techniques (PST). With proper integration of GMMs into the already existing control measures employed in the management of mosquitoes, a remarkable decrease in the prevalence of malaria is envisaged.
\end{abstract}

Keywords: Bioengineering; Genetically modified mosquitoes (GMMs); Population replacement technique (PRT); Population suppression techniques (PST).

\section{Introduction}

One of the major threat to the existence of man on earth is the proliferation of diseases. Diseases can be communicable and non-communicable in
Received

December 24, 2018

Accepted

February 23, 2019

Released April 30, 2019

Full Text Article

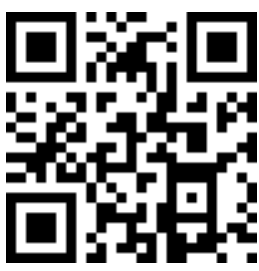

ORCID
0000-0002-3106-4328
Kayode David Ileke
0000-0001-5533-4970
Isaac Omotayo
Olabimi 
and this is so because an outbreak of a communicable disease is usually associated with high morbidity and mortality rate. Communicable diseases can be transmitted through contact, food, vector etc. The proliferation of communicable diseases is high in the tropical region of the world that are affected by issues of overcrowding and poverty (WHO, 2007). This is so because countries that fall in this region are impoverished due to poverty and face a lot of problems associated with vectorborne diseases due to the fact that the weather conditions of their region favour the blossoming populations of these vectors. Mosquitoes are on the front burner in the league of vectors because of it vectoring serious disease conditions that are associated with high rate of illness and death (WHO, 2001). Out of the disease transmitted by mosquitoes, malaria is a serious problem because it has the highest morbidity and mortality rate among vectored diseases (WHO, 2015). Various methods have been employed by man to curb this menace associated with mosquito-borne diseases. It is generally believed that elimination of the vectors will go a long way to as breaking the chain of transmission of the diseases they transmit and also the development of effective healthcare facilities to carter for people already being afflicted with the disease. Several control measures have been employed for taming the population of mosquitoes, the range from the use of chemical, biological and physical means and the prominent of all the previously mentioned control measures is the use of chemical means which is the most commonly used method of control. Use of chemicals has their disadvantages especially use of chemicals that are persistent (high residual effect) in nature and not easily degraded in the ecosystem with some insect even developing resistance. Many non-governmental organisations (NGOs) such as the United Nations (UN), World Health Organization (WHO), United Nations Development
Programme (UNDP), United Nations Children's Fund (UNICEF), World Bank among others have developed and executed a lot of intervention plans aimed at curbing mosquito-borne diseases especially malaria. Some of the programs focus on distribution of insecticidal net, testing and treatment of the mosquito-borne diseases and provision of funds for impoverished countries to support their own efforts in combating these diseases. Currently, the use of insecticidal treated nets (ITNs) and indoor residual spraying (IRS) are considered the mainstay in controlling malaria vectors in sub-Saharan Africa (WHO, 2017). As effective as these control measures tend to be, they have their various shortcomings. Scientists have devised new methods of controlling mosquitoes which are biological in nature to reduce the adverse effect associated with the use of chemicals as a means of control. The use of genetic manipulation of the mosquito genome to render them useless as vectors for diseases is one such method that is showing promise of late.

\section{Mosquito control measures}

Right from the discovery of mosquitoes as being a pest in terms of its ability to cause grievous health hazards to man, several control measures have been devised by man. The earliest effort was the use of habitat modification which involved the use of environmental sanitation to make the environment unfit for the multiplication of mosquitoes (WHO, 2001).

Chemical means were developed and this feat greatly helped to control them as insecticides such as dichlorodiphenyl-trichloroethane (DDT) were at the front burners as the choice chemical for control (Najera et al., 2011). However, DDT lost its effectiveness due to the development of resistance by mosquitoes to it and this led to the development of more effective chemicals to be used as insecticides. The major 
problem associated with the use of chemicals is their potential of being toxic to humans if care is not taken (Barnes, 1953).

So because of the problem associated with chemical use, scientist have been prompted to develop control measures that are eco-friendly. These methods include the use of biological means such as the use of guppies and Bacillus thuriengiensis var israelensis to parasitize larvae of mosquitoes, genetic modification of the mosquitoes to control their population so as to reduce their numbers significantly to the levels whereby they would no longer constitute a nuisance to humans (Gabriel et al., 2014). Other methods include the use of screens and nets to prevent the entry of the mosquitoes into man's habitation. The major method the WHO employ in reducing the incidence of malaria is the use of insecticidal treated nets (ITNs) and indoor residual spraying (IRS) in order to deter the vectors from having access to bite man (WHO, 2017).

\section{Malaria parasites and vector}

Malaria result from the introduction of the Plasmodium parasite (a protozoan) into the human body by female Anopheles mosquitoes. This parasite introduction triggers a series of actions that lead to the manifestation of malaria fever. Only four species of Plasmodium as of today are known to be responsible for human malaria and they are $P$. falciparum, $P$. vivax, $P$. malariae and $P$. ovale (WHO, 2007). However, there have been reports of non-human malaria parasite species specifically $P$. knowlesi infecting humans in SouthEast Asia with increasing frequency that is alarming, but there is no sufficient evidence to account for human-to-human transmission (WHO, 2007). Out of the human malaria parasite species, $P$. falciparum and $P$. vivax pose the greatest threat to humans in terms of the severity of their malaria manifestations in humans. $P$. falciparum is categorized as the most dangerous of them all and is responsible for the majority of malariarelated deaths (WHO, 2017). P. vivax malaria accounts for about half of malaria cases outside Sub-Saharan Africa, and prevails in countries that are prime candidates for elimination; the parasite is said to be responsible for more than $70 \%$ of malaria cases in countries with fewer than 5000 cases each year (WHO, 2015). P. vivax manifestation is different from $P$. falciparum, which does not present itself as a persistent liver-stage infection because it can stay inactive in the liver for many months or even years after inoculation and can cause repeated relapses, $P$. vivax. This has made the elimination of $P$. vivax malaria to be challenging and may in some situations require new tools and strategies to be employed (WHO, 2015).

There are about 515 Anopheles species have been recorded, and out of which only 30-40 are considered important malaria vectors (WHO, 2017). Diverse species can exist within a specific location, with each of them having their different biting and resting pattern and host preference thus, species differ in their transmission efficiency and in their susceptibility to existing or potential antimosquito interventions (WHO, 2017).

\section{Bioengineering mosquitoes to stop their disease transmission capabi- lities}

Genetic engineering of mosquitoes has the potential to be an effective control measure for curbing mosquito-borne diseases (Catteruccia, 2007). This technique provides the opportunity to create designer mosquito strains so as to control their population thereby limiting their capability of diseases transmission (Gabriel et al., 2014) and this can be implemented as population replacement and suppression. These modified mosquitoes can be referred to as 'transgenic mosquitoes' 
and they are developed using genetic tools to alter the mosquito genome.

\section{Population replacement tech- niques (PRT)}

This population replacement technique is based on two integral components according to Wilke et al. (2009) which are a mechanism for resistance and a method to spread the anti-pathogenic gene into the population. The technique utilizes the use of a special genetic system that is capable of spreading the choice anti-pathogenic gene through a targeted vector population (Marrelli et al., 2006). Several mosquito species have been modified to be pathogen resistant, examples are the use of artificial peptides (SM1) to inhibit the development of Plasmodium species in Anopheles stephensi (Ito et al., 2002), expression of cecropin in Anopheles gambiae to impair Plasmodium species development in (Kim et al., 2004) and RNAi (Ribonucleic Acid i) to minimize the transmission of dengue fever in Aedes aegypti (Franz et al., 2006). Since mosquito-borne diseases are transmitted when the mosquito ingested infected blood meal, the ingestion leads to the activation of many genes encoding digestive enzymes (Moreira et al., 2000). Specific regions of the genes known as regulatory regions of those gene type can be used to produce anti-pathogenic effectors molecules in modified vectors in a defined temporal and spatial manner tailored towards maximally affecting a pathogen in a way that it becomes less effective in causing diseases (Kokoza et al., 2001). PRT a self-sustaining one as it requires the introduction of modified mosquitoes once or few times as it will increase its population on its own thereby keeping it at high frequency by releasing only a few numbers of them at a time (WHO, 2009).

\section{genic strain \\ Development of anti-patho- \\ The expression of exogenous genes via the transposon-mediated}

integration of transgenes was the first genomic technology to be developed in mosquitoes, and this gave birth to the modern field of mosquito genome engineering (Coates et al., 1998; Grossman et al., 2001). At the outset, different exogenous 'effector' genetic elements are cloned between the transposon terminal repeats (Grossman et al, 2001) to form a synthetic element that in the presence of the integrating enzyme transposase, inserts into the mosquito genome at quasi-random loci. In order to identify successful transformants, synthetic transposons are generally designed to carry a fluorescent reporter construct, such as the green fluorescent protein (GFP) acting as a selectable marker (Chalfie et al, 1994) that will enable easy detection. This system can be used to incorporate antipathogenic properties into the mosquitoes to render them incapable of disease transmission. The fat body of mosquito is targeted for the engineering of anti-pathogenic properties reasons being that it is the most notable secretory tissue that releases its product to the haemolymph which is a major pathway for most pathogens (Kokoza et al., 2001). Hence, these anti-pathogenic mosquitoes are employed to replace the population of indigent mosquito species.

\section{Gene drives employed in PRT}

Several gene drives have been employed in making mosquito antipathogenic leading the population replacement and the most used ones are:

\section{(HEGs)}

Homing endonuclease genes

Homing endonuclease genes are highly specific Deoxyribonucleic acid (DNA) endonucleases observed in some viruses, bacteria and eukaryotes (Chevalier and Stoddard, 2001). These genes are characterized by 'selfish' genetic elements that have a nonmendelian inheritance in the sense that they are capable of spreading in a 
population irrespective of the fact that they have no beneficial use to the host organism (Gabriel et al., 2014). These genes have been anticipated to alter wild-type mosquito population. The endonuclease stimulates the movement of its encoding DNA from one allele to the other by creating a double-strand break (DSB) at a specific, long (15-40 bp) target site in an allele that lacks the HEG and the homologous DNA repair before copying the HEG to the cut chromosome in a process called 'gene conversion (Chevalier and Stoddard, 2001; Volna et al., 2007). HEGs can be employed in disrupting genes that give mosquito the capability to vector Plasmodium species or push a recombinant refractory gene through a population that is designed to render the mosquito incapable of transmitting the malaria parasites (Gabriel et al., 2014). It could also be used to reduce the population of mosquitoes by targeting female genes that are essential to female fertility thereby reducing her fecundity (Hahn and Nuzhdin, 2004). HEGs could also target $\mathrm{X}$-linked sequences that are expressed during male spermatogenesis from the Y chromosome to avidly tilt the sex ratio of the population towards the abundance of more males at the expense of their female counterpart which will be reduced (Hahn and Nuzhdin, 2004).

\section{Zinc finger nucleases (ZFNs)}

Zinc finger nuclease (ZFN) technique is a genome editing method that is based on the specificity of DNA binding to the zinc finger nuclease. It is a target-specific that is engineered to bind and cleave with DNA at needed sites of the genome (Reegan, et al., 2016). The system comprises of DNA binding domain in association with zinc finger which capable of recognising the specific sequence on the genome and nuclease domain made up of FokI enzyme that cleaves the specific site of the DNA (Reegan et al., 2016). The DNA binding domain of individual ZFNs characteristically is made up of three to six individual zinc finger repeats with each finger capable of recognizing three base pairs. With the use of this procedure, it is possible for ZFN to induce double-stranded breaks (DSB) at a specific section on the genome and with the aid of endogenous DNA repair this technique which was used to modify the genome sequence of higher animals (Kim et al., 1996; Wolfe et al., 2001; Bibikova et al., 2001) could be used to also modify the genome of mosquitoes to make them anti-pathogenic.

\section{Transcription activator-like effector nucleases (TALENs)}

The transcription activator-like effector nucleases (TALEN) is somewhat alike with the Zinc finger nuclease (ZFN) technique only that the former is easier compared to the later and more specific to target sequence (Reyon, 2012). The DNA binding domain of TALEN comprises of a highly conserved repeat of 33-34 amino acid sequence with a difference at the 12th and the 13th amino acids with the two positions exhibiting variation in terms of their positioning and shows a powerful correlation with specific nucleotide recognition in the genome (Reegan, et al., 2016). The relationship existing between amino acid sequence and DNA recognition has enabled the engineering of the specific DNA-binding domains. The possibility of editing a great number of genome has been made easy because of DNA binding domain is fused with the FokI nuclease enzyme which confers extreme site specificity editing (Mussolino and Cathomen, 2012; Sakuma and Woltjen, 2014).

\section{Clustered regulatory inter- spaced short palindromic repeats (CRISPR) and CRISPR associated Protein 9 (Cas9)}

The CRISPR/Cas9 is an RNAguided endonuclease technology that has proven to be a highly efficient tool for making breaks in the genomes of bacteria, yeast, plants and animals 
(Reegan, et al., 2016). The system is much easier and convenient to handle than those of the ZFN and TALEN. The CRISPR/Cas9 technique has been observed to play a similar role to that of an acquired immune system against viruses and phages through CRISPR RNA (crRNA)-guided DNA binding and Cas9 nucleases-mediated DNA breakage in bacteria and archaea (Grissa et al., 2007). Due to fact that CRISPR/Cas9 system behaves like viruses in terms of their behaviour, they tend to attack the ookinete of the malaria pathogen in the midgut of the female Anopheles mosquito rendering it incapable of transmission of malaria. In genome editing, CRISPR/Cas9 system operates with the aid of the single guide RNA (sgRNA) capable of recognizing the target sequence (protospacer) in the genome of host organism through complementary base pairing after which the Cas9 nuclease specifically makes a double-stranded break (DSB) at a region near to the Protospacer Adjacent Motif (PAM) sequence (Jinek et al., 2012).

It should be known that all the above-mentioned gene drive techniques could be useful in creating strains of female Anopheles mosquitoes with refractory or anti-pathogenic characteristics before using them to replace the local population of the mosquitoes.

\section{Population suppression techni- ques (PST)}

Population suppression techniques can be employed in controlling the proliferation of Anopheles mosquito species by introducing modified ones into the wild population which will help effectively reduce their population below the threshold of them being a menace thereby reducing their potentials of spreading the malaria parasite. The latest method currently in use is a technology referred to as 'release of insects carrying a dominant lethal gene' (RIDL) (Alphey, 2002) which is based on sterile insect technique (SIT) (Knipling, 1955) a foremost method employed in population suppression of insects. Using this RIDL, the transgenic male mosquitoes are released into the wild to mate with the wild females so that the resulting offspring will inherit the lethal gene which will invariably kill them, thereby reducing their population below the threshold required for them to constitute disease transmission threatening status (Alphey, 2002; Marrelli et al., 2006; WHO, 2009). However, PST is self-limiting and not sustainable due to the fact that it requires a constant introduction of large numbers of sterile males into the population which is usually capital intensive (WHO, 2009).

\section{Development of sterile strains}

SIT was the first transposonbased technology used to create mosquito strains that are tailored towards the suppression or even elimination of mosquito population (Knipling, 1955). SIT technique uses radiation to induce dominant lethal mutation in the irradiated sperm such that most eggs die soon after fertilization by such sperm (Alphey et al., 2013). This irradiation of the insect help achieve higher sterility but its downside is that it may damage the insect in such a way that their fitness may be affected (Barclay, 2005) making it imperative to modify the technique. A technique known as incompatible insect technique (IIT) which made use of Wolbachia bacteria to induce a form of sterility known as Cytoplasmic incompatibility (CI), whereby the embryo produced by an uninfected females that were fertilized by infected males was developed to solve this irradiation related problem (Brelsfoard et al., 2008). RIDL modification is an improvement on SIT (Phuc et al., 2007). Its primary aim is to render the male sterile by introducing a gene that is lethal to the survivor of offspring of the wild female mosquitoes they mate with, especially the late larval 
stage after hatching from the egg. The system utilizes a gene encoding the tetracycline transactivator (tTA) protein under the control of the tetracyclineresponsive element (tRE) and the Binding of tetracycline to tTA prevents tTA from activating transcription; when tetracycline is removed, tTA instead binds to tRE, thereby inducing its own expression via a positive feedback loop (Gabrieli et al., 2014). The accumulation of tTA is toxic to cells and ultimately leads to organismal death.

\section{Methods used in PST}

The methods that are currently being used in population suppression of mosquito species include:

Sterile insect technique (SIT). The sterile insect technique is a technique that employs the use of radiation in the laboratory to render adult insect (especially males) sterile before releasing them into the wild after which they mate with other wild species to produce non-viable offspring thereby controlling the population of the insect (Reiter, 2007). This technique could be used in controlling the population of mosquitoes so that they would not cause man any discomfort in terms of disease transmission if large numbers of sterile males are released into the wild (Knipling, 1955). The SIT does not cause any ecosystem disruption because it does not introduce any mutation into the natural population and it does not have any effect on non-targeted organisms (Hendrichs et al., 2002). The problem of SIT is that the use of radiation as a sterilizing agent come at a cost to the mosquitoes in the sense the males are frail making lack strength in competing for a mate (Atkinson and Michel, 2002). For high effectiveness of SIT, sterilizing and releasing only male mosquitoes into the wild are useful for achieving the desired result (Alphey, 2002; Fu et al., 2010).

\section{Insect incompatibility techni-}

que (IIT). Insect incompatibility technique is synonymous to SIT. The major difference the two techniques is that IIT uses certain symbiotic bacteria as sterilization agent rather than the radiation used by SIT (Alphey et al., 2013). The bacteria are genetically modified before being introduced inside the mosquito to express effector molecule within it (Beard et al., 1992 and 1993). The bacteria to be used for this procedure must have an effector molecule that will achieve the desired effect, a mechanism to display the effector molecule on the surface of the bacteria, ability of the bacteria to survive long enough to produce the number of required effector to achieve desired result and must not be pathogenic to humans (Riehle et al., 2005 and 2007). In the midgut of the mosquitoes, the use of genetically modified bacteria inhibits blood digestion by displacing the naturally occurring commensal bacteria found in the midgut of the mosquito thereby reducing fecundity which consequentially inhibits the proliferation of mosquito species and then reduces the spread of mosquito-borne diseases (Gajo et al., 2011). Example of such bacteria is the use of genetically modified Wolbachia species a gram -ve bacteria which is highly effective in delivering effector molecules within the mosquito (Yoshida et al., 2001).

Release of insects carrying a dominant lethal gene (RIDL). The Release of Insects Carrying a Dominant Lethal Gene (RIDL) is an improvement on the sterile insect technique. Instead of using radiation to render the mosquito sterile as used in the SIT, a lethal dominant gene which could be controlled by a female-specific promoter could be introduced into the mosquitoes to render them sterile (Gabriel et al., 2014). A colony of mosquito can be maintained by the expression of the lethal gene which could be inactivated by tetracycline treatment where the removal of 
tetracycline from the system could help separate male from female whenever a separation is needed (Wilke and Marrelli, 2012). This system is dependent on tTA expression which is a fusion protein that combines sequence-specific tetracyclinerepressible binding to tRe, a tetracyclineresponse element, to a eukaryotic transcriptional activator (Wilke and Marrelli, 2012). tTA in the absence of tetracycline will bind with the tRe sequence which will in turn activate transcription under the influence of a nearby minimal promoter resulting in the production of a lethal gene. During preparation of mosquitoes for release, the lethal gene is expressed by inactivating the repressor and this will result in the death of all females (Wilke and Marrelli, 2012). The homozygous males carrying the lethal gene are then released into the wild to mate with females which will eventually result in the production of heterozygous offspring in which only the males are spared and the females killed leading to suppression of the local population of the mosquitoes (Heinrich et al., 2000; Thomas et al., 2000). Releasing of Insects carrying a Dominant Lethal gene helps to solve the problem associated with SIT because the mosquitoes produced using RIDL techniques have more strength in terms of competing with mate compared to the SIT whereby the insects are frail and do not have the vigour to compete for a mate (Wyss, 2000). Some systems of RIDL that act at late developmental stage could help control mosquito population by preventing them from maturing into an adult which is the harmful stage of mosquito (Thomas et al., 2000). Also, the female version makes use of the release of homozygous females and withdrawing the repressor to kill all males or a combination of a bisex-lethal system with female lethality to kill all sexes (Wilke and Marrelli, 2012).

\section{Challenges Using GMMs}

The use of genetically modified mosquito has the potential of being the mainstay in the control of malaria through the control of the anopheline mosquitos. However, it is faced with various challenges and these challenges are as follows:

\section{Financial challenges}

Due to the complexities of the method involved in the design and production of transgenic mosquitoes, a huge financial outlay is required for training and equipping of personnel, production and deployment of modified mosquitoes. Hence, there is need to get appropriate means of funding to ensure that the deployment of these mosquitoes is hitch free.

\section{Technical challenges}

Certain aspects of the mosquito behaviour must be understood before genetically altering them to be deployed as a means of control. Understanding the biology of the mosquitoes because vector control is tailored towards controlling the female mosquitoes because they are majorly responsible for disease transmission (Macer, 2003). Hence, the release of male mosquitoes to act as control mechanism for the female is considered to be more logical because releasing of the female will be problematic because their source of food is humans from whence they obtain blood as compared to the males that have their food source readily available in the form of plants from where they obtain nectar. This implies that method to be used must ensure that it does not affect the fitness of the males to enable them effectively compete with the wild males for a mate (Knols et al, 2007). Mating behaviour is another key element that needs to be studied. This is because 
some species of mosquitoes mate multiple times which means that the male must be designed in such a way that they attract female mates for multiple copulations to achieve maximum results. Thirdly, a good knowledge of the colonization and mass production effect of mosquito is required. Colonization has to do with the habits of the mosquito as some are found indoors and others stay outdoors which will enable the gene required to achieve maximum control measure will be properly dispersed among the local mosquito population making monitoring of the gene flow. The mass production effect comes into play in such a way that all modified mosquitoes that pass through the same production technique to have the same characteristics irrespective of the fact that they were mass produced (Knols et al., 2007). Both colonization and mass production effect is essential in fashioning quality control measures. Finally, the biology plays a vital role in ensuring the success or failure of any control measures because of the local population dynamics might affect the effectiveness of genetic control measures applied.

\section{Social ethical and legal challenges}

Since the inception of the use of genetically modified organisms (GMO), some rights groups have been advocating that such a trend should be discontinued. This is because they feel that such use of GMOs would alter the biodiversity of the ecosystem and would infringe on peoples fundamental rights. Genetic methods are focused on modifying the insect in such a way that it will deviate from its natural form as a result of the alteration of its genome. These rights groups also emphasize on obeying animal rights stating that animals also have basic rights which must be broken irrespective of man's selfish nature to make himself comfortable at the detriments of animals (Macer, 2003). The human rights side of things against the use of GMMs in controlling disease transmission especially malaria is that it could pose a public health risk in the sense that these mutant mosquitoes might even gain the ability to transmit even deadlier pathogens to man. To this effect, it beckons on expert to device a standardized approach and best practices that are in tandem with the Convention on Biological Diversity (CBD)/ Cartegena protocol regulation on the use of GMOs and integration of enduser concerns throughout biosafety process through proper consultation with the end users to allay their fears concerning the deployment of such modified mosquitoes (WHO, 2009). Proper risk assessment should be carried out before the use of these GMMs to mitigate against any risk that the ecosystem might be faced with. Before the deployment of GMMs in an area, proper legal permits must be obtained from appropriate bodies (Oxitec, 2017). The processes involved in getting these permits are quite cumbersome and this might frustrate the use of GMMs as a control technique.

\section{Conclusion}

The use of GMMs has the potential of being an effective tool in the control of mosquito population which will in turn transform to the control of mosquito-borne diseases especially malaria as the leading company in the production of GMMs Oxitec has recorded success in their test trials in the use of GMMs in controlling local mosquito population in Cayman Islands, Panama and Brazil. However, should not be seen as a magic bullet i.e. the super control measures as the use of GMMs method still require a great deal of work to be done as researches are still at their various trial stages. Genetically modified mosquito should be integrated into already existing control measures (use of insecticidal nets and Indoor residual spraying). People should not be scared over the deployment of GMMs around 
them as they have not been proven to cause any deleterious health effects because mostly the male mosquitoes are deployed and they do not feed on human's blood rather they feed on nectar.

\section{Conflict of interest}

Authors declare that they have no conflict of interests.

\section{References}

Alphey, L. Re-engineering the sterile insect technique. Insect Biochemistry and Molecular Biology, v. 32, p. 1243-1247, 2002.

Alphey, L.; Andrew, M.; Derric N.; Marco, N. O.; Renaud, L.; Kelly, M.; Camilla, B. Genetic control of Aedes mosquitoes. Pathogen and Global Health, v. 107, no. 4, p. 170-179, 2013.

Atkinson, P. W.; Michel, K. What's buzzing? Mosquito genomics and transgenic mosquitoes. Genesis, v. 32, p. 42-48, 2002.

Barclay, H. J. Mathematical models for the use of sterile insects. Dyck, V. A.; Hendrichs, J.; Robinson, A.S. (Eds.). Sterile insect technique: Principles and practice in areawide integrated pest management. New York: Springer, 2005. p. 147-174.

Barnes, J. M. Toxic hazards of pesticides. Bulletin World Health Organization, v. 8, p. 419-490, 1953.

Beard, C. B.; Mason, P. W.; Aksoy, S.; Tesh, R. B.; Richards, F. F. Transformation of an insect symbiont and expression of a foreign gene in the Chagas disease vector Rhodnius prolixus. American Journal of Tropical Medicine Hygiene, v. 46, p. 195-200, 1992.

Beard, C. B.; O'Neill, S. L.; Tesh, R. B.; Richards, F. F.; Aksoy, S. Modification of arthropod vector competence via symbiotic bacteria. Parasitology Today, v. 9, p. 179183, 1993.

Bibikova, M.; Carroll, D.; Segal, D. J.; Trautman, J. K.; Smith, J.; Kim, Y. G.; Chandrasegaran, S. Stimulation of homologous recombination through targeted cleavage by chimeric nucleases. Molecular and Cell Biology, v. 21, p. 289-297, 2001.
Brelsfoard, C. L.; Sechan, Y.; Dobson, S. L. Interspecific hybridization yields strategy for South Pacific filariasis vector elimination. Public Library of Science Neglected Tropical Diseases, v. 2, e. 129, 2008.

Catteruccia, F. Malaria vector control in the third millennium: progress and perspectives of molecular approaches. Pest Management Science, v. 63, p. 634-640, 2007.

Chalfie, M.; Tu, Y.; Euskirchen, G.; Ward, W. W.; Prasher, D. C. Green fluorescent protein as a marker for gene expression. Science, v. 263, p. 802-805, 1994.

Chevalier, B. S.; Stoddard, B. L. Homing endonucleases: Structural and functional insight into the catalysts of intron/intein mobility. Nucleic Acid Research, v. 29, p. 3757-3774, 2001.

Coates, C. J.; Jasinskiene, N.; Miyashiro, L.; James, A. A. Mariner transposition and transformation of the yellow fever mosquito, Aedes aegypti. Proceedings of the National Academy of Sciences USA, v. 95, p. 37483751, 1998.

Franz, A. W.; Sanchez-Vargas, I.; Adelman, Z. N.; Blair, C. D.; Beaty, B. J.; James, A. A.; Olson, K. E. Engineering RNA interferencebased resistance to dengue virus type 2 in genetically modified Aedes aegypti. Proceedings of the National Academy of Sciences USA, v. 103, p. 4198-4203, 2006.

Fu, G.; Lees, R. S.; Nimmo, D.; Aw, D.; Jin, L.; Gray, P. Female-specific flightless phenotype for mosquito control. Proceedings of the National Academy of Sciences USA, v. 107, p. $4550-4554,2010$.

Gabrieli, P.; Smidler, A.; Catteruccia, F. Engineering the control of mosquito-borne infectious diseases. Genome Biology, v. 15, p. 535, 2014.

Gaio, A. O.; Gusmão, D. S.; Santos, A. V.; Berbert-Molina, M. A.; Pimenta P. F.; Lemos F. J. Contribution of midgut bacteria to blood digestion and egg production in Aedes aegypti (Diptera: culicidae). Parasites and Vectors, v. 14, p. 94-105, 2011.

Grissa, I.; Vergnaud, G.; Pourcel, C. The CRISPRdb database and tools to display CRISPRs and to generate dictionaries of spacers and repeats. BMC Bioinformatics, v.8, p. 1-10, 2007. 
Grossman, G. L.; Rafferty, C. S.; Clayton, J. R.; Stevens, T. K.; Mukabayire, O.; Benedict M. Q Germline transformation of the malaria vector, Anopheles gambiae, with the piggy Bac transposable element. Insect Molecular Biology, v. 10, p. 597-604, 2001.

Hahn, M. W.; Nuzhdin, S. V. The fixation of malaria refractoriness in mosquitoes. Current Biology, v. 14, p. 264-265, 2004.

Heinrich, J. L.; Scott, M. J. A repressible female-specific lethal genetic system for making transgenic insect strains suitable for a sterile-release program. Proceedings of the National Academy of Sciences USA, v. 97, p. 8229-8232, 2000.

Hendrichs, J.; Robinson, A. S.; Cayol, J. P.; Enkerlin, W. Medfly areawide sterile insect technique programmes for prevention, suppression or eradication: the importance of mating behavior studies. Florida Entomology, v. 85, p. 1-13, 2002.

Ito, J.; Ghosh, A.; Moreira, L. A.; Wimmer, E. A.; Jacobs-Lorena, M. Transgenic anopheline mosquitoes impaired in transmission of a malaria parasite. Nature, v. 417, p. 452-455, 2002.

Jinek, M.; Chylinski, K.; Fonfara, I.; Hauer, M.; Doudna, J. A.; Charpentier, E. A programmable dual-RNA-guided DNA endonuclease in adaptive bacterial immunity. Science, v. 337, p. 816-821, 2012.

John, W.; Kathy, L.; Scot, L.; Sherryl, T.; Marcia, J. G. A Monthly Bulletin on Epidemiology and Public Health Practice in Washington. epiTrends, v. 20, p. 3, 2015.

Kim, W.; Koo, H.; Richman, A. M.; Seeley, D.; Vizioli, J.; Klocko, A. D.; O’Brochta, D. A. Ectopic expression of a cecropin transgene in the human malaria vector mosquito Anopheles gambiae (Diptera: Culicidae): effects on susceptibility to Plasmodium. Journal of Medical Entomology, v. 41, p. 447-455, 2004.

Kim, Y. G.; Cha, J; Chandrasegaran, S. Hybrid restriction enzymes: Zinc finger fusions to Fok I cleavage domain. Proceedings of the National Academy of Sciences USA, v. 93, p. 1156-1160, 1996.

Knipling, E. F. Possibilities of insect control or eradication through the use of sexually sterile males. Journal of Economic Entomology, v. 48, p. 902-904, 1995.
Knols, B. G. J.; Bossin, H. C.; Mukabana, W. R.; Robinson, A. S. Transgenic mosquitoes and the fight against malaria: Managing technology push in a turbulent GMO world. America Journal of Tropical Medicine and Hygiene, v. 77, no. 6, p. 232-242, 2007.

Kokoza, V.; Ahmed, A.; Wimmer, E. A.; Raikhel, A. S. Efficient transformation of the yellow fever mosquito Aedes aegypti using the piggyBac transposable element vector pBac[3xP3-EGFP afm]. Insect Biochemical and Molecular Biology, v. 31, p. 1137-1143, 2001.

Macer, D. R. J. Ethical, legal and social issues of genetically modified disease vectors in public health. Geneva: UNDP/World Bank/WHO, 2003.

Marrelli, M. T.; Moreira, C. K.; Kelly, D.; Alphey, L.; Jacobs-Lorena, M. Mosquito transgenesis: what is the fitness cost? Trends in Parasitology, v. 22, p. 197-202, 2006.

Moreira, L. A; Edwards, M. J.; Adhami, F.; Jasinskiene, N.; James, A. A.; Jacobs-Lorena, M. Robust gut-specific gene expression in transgenic Aedes aegypti mosquitoes. Proceedings of the National Academy of Sciences USA, v. 97, p. 10895-10898, 2000.

Mussolino, C.; Cathomen, T. TALE nucleases: Tailored genome engineering made easy. Current Opinion in Biotechnology, v. 23, p. 644-650, 2012.

Najera, J. A.; Gonzalez-Silva, M; Alonso, P. L. Some lessons for the future from the global malaria eradication programme (19551969). Public Library of Science Medicine, v. 8, no. 1, e. $1000412,2011$.

Oxitec. Oxitec's Genetically Modified Mosquitoes: Ready to roll out?. Gene watch UK. 2017.

Phuc, H. K.; Andreasen, M. H.; Burton, R. S.; Vass, C.; Epton, M. J.; Pape, G.; Fu, G.; Condon, K. C.; Scaife, S.; Donnelly, C. A.; Coleman, P. G.; White-Cooper, H.; Alphey, L. Late-acting dominant lethal genetic systems and mosquito control. BMC Bioinformatics, v. 5, p. 11, 2007.

Reegan, D. A.; Ceasar, A. S.; Paulraj, G. M.; Ignacimuthu, S.; Al-Dhabi N. A. Current status of genome editing in vector mosquitoes: A review. Bioscience Trends, p. 1-9, 2016. 
Reiter, P. Oviposition, dispersal and survival in Aedes aegypti: implications for the efficacy of control strategies. Vector-Borne Zoonotic Diseases, v. 7, p. 261-73, 2007.

Reyon, D.; Tsai, S. Q.; Khayter, C.; Foden, J. A.; Sander J. D.; Joung J. K. FLASH assembly of TALENs for high-throughput genome editing. Nature Biotechnology, v. 30, p. 460-465, 2012.

Riehle, M. A.; Jacobs-Lorena, M. Using bacteria to express and display anti-parasite molecules in mosquitoes: current and future strategies. Insect Biochemistry and Molecular Biology, v. 35, p. 699-707, 2005.

Riehle, M. A.; Moreira, C. K.; Lampe, D.; Lauzon, C.; Jacobs-Lorena, M. Using bacteria to express and display anti-Plasmodium molecules in the mosquito midgut. International Journal of Parasitology, v. 37, p. 595-603, 2007.

Sakuma, T.; Woltjen, K. Nuclease-mediated genome editing: At the front-line of functional genomics technology. Development Growth and Differentiation, v. 56, p. 2-13, 2014.

Thomas, D. D.; Donnelly, C. A.; Wood, R. J.; Alphey, L. S. Insect population control using a dominant, repressible, lethal genetic system. Science, v. 287, p. 2474-2476, 2000.

Volna, P.; Jarjour, J.; Baxter, S.; Roffler, S. R.; Monnat, J., Jr.; Stoddard, B. L. Flow cytometric analysis of DNA binding and cleavage by cell surface-displayed homing endonucleases. Nucleic Acid Research, v. 35, p. 2748-2758, 2007.

WHO - World Health Organization. A framework for malaria elimination. Geneva, Switzerland: WHO, 2017.
WHO - World Health Organization. Achieving the malaria millennium development goal target: Reversing the Incidence of Malaria 2000-2015. Geneva, Switzerland: WHO, 2015.

WHO - World Health Organization. Malaria elimination: A field manual for low and moderate endemic countries. Geneva, Switzerland: WHO, 2007.

WHO - World Health Organization. Progress and prospects for the use of genetically modified mosquitoes to inhibit disease transmission. Geneva, Switzerland: WHO, 2009.

WHO - World Health Organization. Vector control. Geneva, Switzerland: WHO, 2001.

Wilke, A. B.; Gomes, A. C.; Natal, D.; Marrelli, M. T. Control of vector populations using genetically modified mosquitoes. Revista de Saúde Pública, v. 43, p.869-874, 2009.

Wolfe, S. A.; Nekludova, L.; Pabo, C. O. DNA recognition by Cys2His2 zinc finger proteins. Annual Review of Biophysics and Biomolecular Structure, v. 29, p. 183-212, 2001.

Wyss, J. H. Screwworm eradication in the Americas. Annals of the New York Academy of Science, v. 916, p. 186-193, 2000.

Yoshida, S.; Ioka, D.; Matsuoka, H.; Endo, H.; Ishii, A. Bacteria expressing singlechain immunotoxin inhibit malaria parasite development in mosquitoes. Molecular and Biochemical Parasitology, v. 113, p. 89-96, 2001. 